\title{
A universal origin for secondary relaxations in supercooled liquids and structural glasses
}

\author{
Jacob D. Stevenson ${ }^{1}$ and Peter G. Wolynes ${ }^{1}$ \\ Peter G. Wolynes: pwolynes@ucsd.edu \\ ${ }^{1}$ Department of Physics and Department of Chemistry and Biochemistry, Center for Theoretical \\ Biological Physics, University of California at San Diego, La Jolla, California 92093
}

\begin{abstract}
Nearly all glass forming liquids display secondary relaxations, high frequency dynamical modes of structural reconfiguration seemingly distinct from the primary alpha relaxations. We show that accounting for driving force fluctuations and the diversity of reconfiguring shapes in the random first order transition theory yields a novel dynamical process which shares many of the features ascribed to secondary relaxations. While primary relaxation takes place through activated events involving compact regions, secondary relaxation is governed by more ramified, string-like, or percolation-like clusters of particles. These secondary relaxations generate a low free energy tail on the distribution of activation barriers which becomes more prominent with increasing temperature. The activation barrier distributions of the two process merge near the dynamical crossover temperature $T_{c}$, where the secondary process ultimately becomes the dominant mode of structural relaxation. These string-like reconfigurations are seen to smooth the transition at $T_{c}$ between high temperature collisional dynamics and activated events.
\end{abstract}

Diversity, a key feature of glassy systems, is most apparent in their relaxation properties. Dielectric, mechanical and calorimetric responses of supercooled liquids are not single exponentials in time, but manifest a distribution of relaxation times. The typical relaxation time grows upon cooling the liquid until it exceeds the preparation time, yielding a nonequilibrium glass, which can still relax but in an age dependent fashion. In addition to the main relaxations that are responsible for the glass transition, supercooled liquids and structural glasses exhibit faster motions, some distinct enough in time scale from the typical relaxation to be called "secondary" relaxation processes[1-5]. These faster motions account for only a fraction of the relaxation amplitude in the liquid but become dominant features in the relaxation of otherwise frozen glass, where they are important to the mechanical properties. These secondary relaxation processes in the solvation shell of proteins are also prominent in protein dynamics[6].

The phenomenology of secondary relaxation has been much discussed but, owing especially to the problem of how to subtract the main peak, the patterns observed seem to be more

Users may view, print, copy, download and text and data- mine the content in such documents, for the purposes of academic research, subject always to the full Conditions of use: http://www.nature.com/authors/editorial_policies/license.html\#terms

Correspondence and requests for materials should be addressed to PGW.

The authors together conceived and implemented the theory and wrote the paper. JDS implemented the numerical work. 
complex and system specific than those for the primary glassy relaxation. Some of the secondary relaxation motions are, doubtless, chemically specific, occurring on the shortest length scales. Nevertheless the presence of secondary relaxation in glassy systems is nearly universal[7]. In this paper we will show how secondary relaxations due to collective particle motion naturally arise in the random first order transition (RFOT) theory of glasses[8] and are predicted to scale in intensity and frequency in a manner consistent with observation.

The RFOT theory is based on the notion that there is a diversity of locally frozen free energy minima that can inter-convert via activated transitions. The inter-conversions are driven by an extensive configurational entropy. RFOT theory accounts for the well known correlations in supercooled liquids between the primary relaxation time scale (characterized by kinetic fragility) and thermodynamics (characterized by the configurational heat capacity) $[9,10]$ as well as the aging behavior in the glassy state[11]. By taking account of local fluctuations in the driving force, RFOT theory also gives a good account of the breadth of the rate distribution of the main relaxation[12,13]. Here we will argue that RFOT theory suggests, universally, a secondary relaxation also will appear and that its intensity and shape depend on the configurational thermodynamics of the liquid. This relaxation generates a low free energy tail on the activation barrier distribution which emerges because of differences in the geometry of the the reconfiguring regions. Near to the laboratory $T_{g}$ the primary relaxation process involves reconfiguring a rather compact cluster, but the reconfiguring clusters become more ramified as the temperature is raised, eventually resembling percolation clusters or strings near the dynamical crossover to mode coupling behavior at $T_{c}$, identified with the onset of non-activated motions[14]. Reconfiguration events of the more extended type are more susceptible to fluctuations in the local driving force, even away from the crossover. These ramified or "stringy" reconfiguration events thus dominate the low barrier tail of the activation energy distribution.

When the shape distribution of reconfiguration processes is accounted for, a simple statistical computation shows that a two peaked distribution of barriers can arise. This calculation motivates a more explicit but approximate theory that gives analytical expressions for the distribution of relaxation times in the tail. In keeping with experiment, the theory predicts the secondary relaxation motions are actually most numerous near the crossover, but of course, merge in frequency with the main relaxation peak in time scale also at that crossover. Furthermore the relaxation time distribution for secondary relaxations is predicted to be described by an asymptotic power law. The theory is easily extended to the aging regime where these secondary relaxations can dominate the rearranging motions.

In RFOT theory, above the glass transition temperature, the entropic advantage of exploring phase space, manifested as a driving force for reconfiguration, is balanced by a mismatch energy at the interface between adjacent metastable states. For a flat interface in the deeply supercooled regime the mismatch energy can be described as a surface tension that can be estimated from the entropic cost of localizing a bead[9, 15], giving a surface tension $\sigma_{0}=(3 / 4) k_{B} T r_{0}^{-2} \ln \left[1 /\left(d_{L}^{2} \pi e\right)\right]$ where $d_{L}$ is the Lindemann length, the magnitude of particle fluctuations necessary to break up a solid structure, and is nearly universally a tenth of the inter-particle spacing, $\left(d_{L}=0.1 r_{0}\right)$. The free energy profile for reconfiguration events 
resembles nucleation theory at first order transitions but is conceptually quite distinct. Following Stevenson-Schmalian-Wolynes (SSW)[14] the free energy cost of an $N$ particle cluster with surface area $\Sigma$ making a structural transition to a new metastable state may be written

$$
F\left(N, \sum\right)=\sum \sigma_{0}-N T s_{c}-k_{B} T \ln \Omega\left(N, \sum\right)-\sum_{\text {particles }} \tilde{f}
$$

A key element of the free energy profile is the shape entropy $k_{B} \log \Omega(N, \Sigma)$ which accounts for the number of distinct ways to construct a cluster of $N$ particles having surface area $\Sigma$. At one extreme are compact, nearly spherical objects with shape entropy close to zero, while objects such as percolation clusters or stringy chains have surface area and shape entropy which both grow linearly with $N$. The last term of equation 1 accounts for the inherent spatial fluctuations in the disordered glassy system. The disorder is represented here as driving force fluctuations such that each particle joining the reconfiguration event is given a random energy, $\tilde{f}$, drawn from a distribution of width $\delta f$. The r.m.s. magnitude of the fluctuations above $T_{g}$ can be thought of as originating primarily from configurational entropy fluctuations and can be estimated as $\delta f \approx T \delta s_{c} \approx T \sqrt{\Delta C_{p} k_{B}}$, a result expected to be valid for large enough regions. For simplicity we will assume no correlations in the fluctuations of the driving force, but such correlations can be included. We presently ignore local fluctuations in the surface mismatch free energy, but their inclusion would not qualitatively alter the results[13, 16, 17].

For nearly spherical reconfiguring regions the shape entropy is negligible and the mismatch free energy term becomes $\sigma_{0} 4 \pi\left(3 N / 4 \pi \rho_{0}\right)^{\theta / 3}$. In disordered systems the mismatch free energies grow with exponent $\theta$ generally less than 2 reflecting the large number of metastable states which can wet the interface and reduce the effective surface tension. A renormalization group treatment of the wetting effect [15] suggests that $\theta=3 / 2$ in the vicinity of an ideal glass transition. Incomplete wetting $(\theta \approx 2)$, potentially applicable for systems with small disorder would not change the numerics of the present theory much. Whether complete wetting occurs for supercooled liquids under laboratory conditions is still debated[17-19]. The free energy profile describing reconfiguration events restricted to compact clusters becomes, then, $F_{\text {compact }}(N)=\sigma_{0} 4 \pi(3 N / 4 \pi \rho 0)^{\theta / 3}-N T s_{c}$. The minimum number of particles participating in a reconfiguration event is determined by finding where the free energy profile crosses zero. For $\theta=3 / 2$ the activation free energy barrier is inversely proportional to the configurational entropy, leading to the Adam-Gibbs[20] relation for the most probable relaxation time $F_{\alpha}^{\ddagger} / k_{B} T \sim \ln \tau_{\alpha} / \tau_{0} \sim s_{c}^{-1}$. Adding fluctuations to the profile of compact reconfiguration events yields an approximate Gaussian distribution of barriers with width scaling as $\sqrt{N \ddagger} \delta f$. Xia and Wolynes[12], and more explicitly Bhattacharyya et al.[21], have shown that, with the inclusion of facilitation effects, the resulting barrier distribution accounts for the stretching of the main relaxation process and yields good estimates for how the stretching exponent varies with liquid fragility. 
Restricting the reconfiguration events to stringy clusters (using percolation clusters gives very similar results), gives a free energy profile linear in the number of particles reconfigured, save for the minimum $\operatorname{cost} F_{\text {in }}$ to begin to reconfigure a region:

$$
F_{\text {string }}(N)=-N T\left(s_{c}-s_{c}^{\text {string }}\right)+F_{\text {in }} .
$$

The critical "entropy" is given by $T s_{c}^{\text {string }}=v_{\text {int }}(z-2)-k_{B} T \ln (z-5) \approx 1.13 k_{B} T$. This is the balance between the surface energy written in terms of the coordination number of the random close packed lattice $z \approx 12$ ( $v_{\text {int }}$ is the surface tension per neighbor and is directly related to $\sigma_{0}$ ) and the shape entropy including excluded volume effects [22]. If a bead can individually reconfigure then the cost to begin to reconfigure is $F_{\text {in }}=z v_{\text {int }}-T s_{c} \approx 3 k_{B} T$. If, more realistically, two must be moved then $F_{\text {in }} \approx 6 k_{B} T$. The continuous form of the surface mismatch energy gives a somewhat higher value when applied to these small regions, giving $F_{\text {in }}^{\text {continuous }}=r_{0}^{2} \sigma_{0} 4 \pi(3 /(4 \pi))^{\theta / 3}-T s_{c} \approx 10 k_{B} T$ for a one particle reconfiguration. The remarkably simple free energy profile of equation 2 monotonically increases, so that below $T_{\text {string }}$ (defined by $s_{c}\left(T_{\text {string }}\right)=s_{c}^{\text {string) }}$ reconfiguration via pure stringy objects is impossible. Above $T_{\text {string }}$ the same process can occur with very small free energy barrier, having only to overcome $F_{\text {in }}$. Thus $T_{\text {string }}$ signals the crossover from dynamics dominated by activated events to dynamics dominated by non-activated processes. This association of $T_{\text {string }}$ with $T_{c}$ is confirmed experimentally [14]. Interestingly, this crossover is mathematically analogous to the Hagedorn transition of particle theory[23]. In contrast to the situation for compact reconfiguration, driving force fluctuations dramatically alter the picture of stringy relaxation. With driving force fluctuations a lucky sequence of fluctuations can easily push the nominally linearly increasing free energy profile to cross zero, enabling this mode of relaxation below $T_{c}$.

SSW[14] introduced a crude model to estimate the shape entropy and surface area for the range of shapes between compact and string-like reconfigurations. This "fuzzy sphere" model represents a reconfiguration event as a compact core of $N_{c}$ particles with a stringy halo of $N_{f}$ particles. With this interpolative model it has been shown that near $T_{g}$ the preferred shape of a reconfiguring region is largely compact, while the relevant regions become more ramified close to $T_{c}$. Disorder, modifying the SSW two dimensional free energy profile, can be implemented by aggregating fluctuations separately in the compact core and in the stringy halo. The local free energy plots for several realizations of such accumulated fluctuations (assumed to have Gaussian statistics) are given in figure 1. For some realizations compact reconfiguration will still be required to overcome the free energy barrier, but for others the fluctuations are such that the free energy cost for reconfiguration crosses zero along the $N_{f}$ axis (shown in the figure in yellow) so the region is able to relax transiently via a string-like reconfiguration event. These stringy rearranging clusters, stabilized by disorder, we argue, are key contributors to the secondary relaxation process.

The statistics of the stable reconfiguration paths with lowest free energy barrier are summarized in figure 2 with barrier distributions for two different values of $\delta f$ corresponding to a strong and a fragile liquid. We can dissagregate these distributions into contributions, 
separately, from compact events and from string-like fluctuation induced events. These distributions are shown in figures 3 and 4 . At temperatures near $T_{g}$ compact primary relaxations dominate reconfiguration, but as the temperature increases, fluctuations are able to stabilize string-like reconfiguration more easily and the secondary relaxations increase in prominence. The units of the activation energy are given in $k_{B} T$ which assumes a mismatch penalty primarily entropic in nature, $\sigma_{0} \sim k_{B} T$. An energetic mismatch penalty, $\sigma_{0} \sim k_{B} T_{K}$, would lead to Arrhenius behavior for the secondary relaxation process because the activation barrier distribution is peaked around the minimum free energy to initiate a stringy reconfiguration, $F_{\text {in }} \sim \sigma_{0}$. Although the secondary peak remains largely stationary, the primary relaxation peak shifts to lower free energy barriers with increasing temperature, a phenomenon which makes distinguishing between the two contributions difficult in experimental data analysis. Figures 3 and 4 make clear that as $T_{c}$ is approached and crossed, the primary and secondary peaks merge and string-like reconfiguration clusters become the dominant mode of activated relaxation. For fragile liquids, i.e. liquids with larger configurational entropy fluctuations [10], the secondary relaxation peak is generally more significant than for strong liquids. Both peaks are broader and begin to merge at lower temperatures for the more fragile liquids. The facilitation phenomenon, as described by Xia and Wolynes[12] and by Bhattacharya et al.[21] but not accounted for here, would shift weight from the largest free energies to the center of the primary peak, raising the overall height of the primary peak relative to the secondary peak. Because facilitation effects are not explicitly accounted for it is not easy directly to compare the predicted distributions with quantitative precision to experiment. In addition, the number of reconfiguring particles in the processes described by the two peaks is different, so their contributions to the measured amplitudes are different as well. Nevertheless, the magnitude of the secondary relaxation peak in the simulation, as compared to the primary peak, is somewhat larger at high temperatures than experiments show, a disparity that is more pronounced for fragile materials. The assumptions in the fuzzy sphere model, and especially the assumption of uncorrelated disorder, apparently overestimate the influence of the fluctuations, which are probably (anti-) correlated for the most fragile systems.

The barrier distribution for reconfiguration events can be analytically calculated by recognizing that with the assumption of uncorrelated disorder equation 1 is a stochastic equation describing a random walk in free energy. This analytic calculation resembles that of Plotkin and Wolynes for the "buffing" of protein folding energy landscapes [26]. Using the random walk analogy allows a full analysis of the distribution of barriers $\Gamma\left(F^{\ddagger}\right)$ for reconfiguration events that take an ideal stringy form. Adding fluctuations to equation 2 and taking the continuous limit leads to a stochastic differential equation for the free energy profile

$$
d F / d N=\varphi+\tilde{f} .
$$

where $\varphi=d F_{\text {string }} / d N=T\left(s_{c}^{\text {string }}-s_{c}\right)$ is the slope of the linear string free energy profile and depends simply on the proximity to the string transition, $\phi$ can be thought of as a string tension reflecting the free energy cost of lengthening a string. The principal quantity to compute in order to ultimately characterize the distribution of barriers is $G\left(N, F ; F_{\text {in }}\right)$, the 
probability that a reconfiguration event involving $N$ particles has free energy cost $F$ if the cost of initiating the event is $F_{\text {in }}$. The evolution of $G\left(N, F ; \tilde{F_{\text {in }}}\right)$ is described by a diffusion equation with drift subject to absorbing boundary conditions at both $F=0$ and $F=F^{\ddagger}$. The upper boundary is associated with the free energy barrier because the absorbing boundaries keep track of the maximum and minimum of the random walk in free energy [27].

The distribution of barriers $\Gamma\left(F^{\ddagger}\right)$ is recovered from $G\left(N, F ; F_{\text {in }}\right)$ through a series of transformations which, in the language of a random walk in free energy, calculate the probability density for the maximum excursion of the path[27].

$$
\Gamma\left(F^{\ddagger}\right)=-\frac{\partial}{\partial F^{\ddagger}} \int_{0}^{N_{\max }} d N\left\langle\left.\frac{\delta f^{2}}{2} \frac{\partial G\left(N, F ; \tilde{F}_{\text {in }}\right)}{\partial F}\right|_{F=0}\right\rangle_{0<\tilde{F}_{\text {in }}<F^{\ddagger}} .
$$

The derivation of this equation is understood by looking at the individual terms. The derivative $-\left.\frac{\delta f^{2}}{2} \frac{\partial G\left(N, F ; \tilde{F}_{\text {in }}\right)}{\partial F}\right|_{F=0}$ yields the probability flow through the lower boundary at step $N$ and is present because we are interested only in nearly stable reconfigurations, i.e. paths that ultimately cross $F=0$. The absorbing boundary condition ensures that we capture only first crossings of the $F=0$ boundary. The absorbing boundary at $F=F^{\ddagger}$ coupled with the derivative $\partial / \partial F^{*}$ captures the statistics of paths which have maximum excursion (reconfigurations which have activation barrier) between $F^{\ddagger}$ and $F^{\ddagger}+\mathrm{d} F^{\ddagger}$. The average $\langle\cdot\rangle_{0<F_{\text {in }}<F} \doteqdot$ acknowledges the fluctuations in the free energy cost of initiating a string, an effect which is not captured in the continuous $N$ random walk. These fluctuations take the form $F_{\text {in }}=F_{\text {in }}+\tilde{f \text { where }} F_{\text {in }}$ and the statistics of the random variable $f$ are described above. The integral in equation 4 sums over contributions from paths of all lengths less than $N_{\max }$, the maximum size to which a stringy reconfiguration event would typically grow before compact reconfigurations dominate. We estimate this maximum length as $N_{\max } \approx F_{\alpha}^{\ddagger} / \varphi$, since certainly by that length the most important reconfiguration events would be compact. For the following calculation of $\Gamma\left(F^{\ddagger}\right)$ we use a smoothed cutoff in which the finite integral $\int_{0}^{N_{\max }} d N$. is replaced by $\int_{0}^{\infty} d N \exp \left(-N / N_{\max }\right)$, which helps to simplify the equations.

The Greens function $G\left(N, F ; \tilde{F_{\text {in }}}\right)$ can be calculated explicitly by solving the diffusion equation using the method of images. The result may be represented in closed form in terms of the Jacobi theta function, however we leave the sum explicit to more easily examine the asymptotics

$$
\begin{aligned}
G\left(N, F ; \tilde{F}_{\text {in }}\right) & =\frac{e^{\frac{\varphi}{\delta f^{2}}\left(F-\tilde{F}_{\text {in }}-\varphi N / 2\right)}}{\sqrt{2 \pi \delta f^{2} N}} \\
& \times \sum_{n=-\infty}^{\infty}\left[e^{-\frac{\left(2 n F^{\ddagger}+F-\tilde{F}_{\text {in }}\right)^{2}}{2 \delta f^{2} N}}-e^{-\frac{\left(2 n F^{\ddagger}+F+\tilde{F}_{\text {in }}\right)^{2}}{2 \delta f^{2} N}}\right]
\end{aligned}
$$

The distribution of free energy barriers follows directly from equations 4 and 5 


$$
\begin{aligned}
\Gamma\left(F^{\ddagger}\right)= & \frac{\partial}{\partial F^{\ddagger}}\left\langle\exp \left(-\frac{F_{\mathrm{in}} \varphi}{\delta f^{2}}-\frac{1}{2} F_{\mathrm{in}} q\right)\right. \\
& \left.\times\left(1-\frac{\exp \left(F_{\mathrm{in}} q\right)-1}{\exp \left(F^{\ddagger} q\right)-1}\right)\right\rangle_{0<F_{\mathrm{in}}<F^{\ddagger}} \\
& \text { where, } q \equiv \frac{2}{\delta f} \sqrt{\frac{2 \varphi}{F_{\alpha}^{\ddagger}}+\frac{\varphi^{2}}{\delta f^{2}}}
\end{aligned}
$$

For the sake of readability the the average and the derivative in the above equation have been left unevaluated, however the full result involves nothing more complicated than exponentials and error functions.

The percent of total structural relaxation which can be attributed to the secondary process (here called $\Psi$ is estimated from the distribution of barriers by calculating the probability that fluctuations can stabilize a stringy reconfiguration for any size barrier less than $F_{\alpha}^{\ddagger}$. Integrating $\Gamma\left(F^{\ddagger}\right)$ over $F^{\ddagger}$ yields

$$
\Psi \approx \exp \left\{-\frac{2 \varphi F_{\text {in }}}{\delta f^{2}}\right\}
$$

It is found that $\Psi$ increases with temperature as the dynamical crossover is approached. This trend is validated experimentally as is demonstrated in figure 6 in which $\Psi$ is directly compared to laboratory data taken from reference [24] for the glass forming liquid toluene. The analytical curve, which has no fitting parameters, accurately predicts the relative importance of the secondary process although the dependence on temperature is slightly stronger than seen experimentally. The theory predicts that at the dynamical crossover temperature and above, this secondary process becomes the only remaining mode of activated relaxation. This change in relaxation mechanism will be seen as a crossover rather than a sharp transition. In fact, the sharp transition from activated to non-activated motions at $T_{c}$ that is predicted by the non-fluctuating RFOT theory, as well as by mode coupling theory [28, 29] and the mean field theory of supercooled liquids[30-32], can be seen as being smoothed out by the string-like activated events, exhibiting no divergent critical behavior[21]. In this temperature regime the non-activated secondary beta relaxations of mode coupling theory would be present and overlap in frequency with the string-like activated secondary relaxations, perhaps making the differentiation of the processes without imaging difficult in the laboratory.

$\Gamma\left(F^{\dagger}\right)$, the distribution of free energy barriers for string-like relaxations, decays exponentially for $F^{\ddagger}>F_{\text {in }}$

$$
\Gamma\left(F^{\ddagger}>F_{\text {in }}\right) \sim \exp \left(-F^{\ddagger} q\right) \approx \exp \left(-2 \frac{F^{\ddagger} \varphi}{\delta f^{2}}\right)
$$

The results agree with the numerically sampled distribution of barriers for the string-like reconfiguration events (shown in figure 5). Using the fact that $\tau=\tau_{0} \exp \left(F^{*} / k_{B} T\right)$, equation 8 gives a power law distribution of relaxation times $P(\tau) \sim \tau^{-\gamma}$ where 
$\gamma \approx 2\left(s_{c}^{\text {string }}-s_{c}\right) / \Delta C_{P}+1$. Well above $T_{g}$ the high barrier side of the secondary relaxation blends in with the primary relaxation peak. Thus the secondary relaxation from ramified reconfiguration events often appears as only a "wing" on the main distribution[33, 34].

In the aging glass, the picture of secondary relaxation is slightly modified. If a cooling liquid falls out of equilibrium at $T_{f}$ (defining $T_{f}$, the fictive temperature) then the frozen-in structure has an average excess energy per particle $\varepsilon\left(T_{f}\right)=\varepsilon\left(T_{K}\right)+\int_{T_{K}}^{T_{f}} d T \Delta C_{P}(T)$. At temperatures $T<T_{f}$ a region of the liquid undergoing reconfiguration would relax to a structure with average energy $\varepsilon(T)<\varepsilon\left(T_{f}\right)$. Thus the driving force for reconfiguration gains an energetic contribution and the configuration entropy in equation 1 is replaced by $T s_{c} \rightarrow\left(T s_{c}+\Delta \varepsilon\right)$ where $\Delta \varepsilon=\varepsilon\left(T_{f}\right)-\varepsilon(T)=\int_{T}^{T_{f}} d T^{\prime} \Delta C_{P}\left(T^{\prime}\right)$. Lubchenko and Wolynes[11] have shown that this additional driving force results in a change in slope of the typical primary relaxation time as a function of temperature and a transition to nearly Arrhenius behavior as the system falls out of equilibrium. Correspondingly, for secondary relaxations, falling out of equilibrium causes the string tension $\phi$ to be reduced by an amount $\Delta \varepsilon$, giving $\varphi=T s_{c}^{\text {string }}=-T s_{c}-\Delta \varepsilon$, making the system appear closer to the dynamical crossover than an equilibrated system at the same temperature. Furthermore, the driving force fluctuations are frozen in as the aging glass falls out of equilibrium and becomes largely independent of temperature. These changes broaden and flatten the barrier distribution as the temperature is lowered, modifying the large free energy barrier decay such that

$$
\Gamma\left(F^{\ddagger}>F_{\text {in }}\right) \sim \exp \left(-2 \frac{F^{\ddagger}\left(T s_{c}^{\text {string }}-T s_{c}-\Delta \varepsilon\right)}{T_{f}^{2} k_{B} \Delta C_{P}\left(T_{f}\right)}\right) .
$$

Similarly, the relative importance to total relaxation of the secondary process in the aging regime becomes

$$
\Psi \approx \exp \left\{-\frac{2\left(T s_{c}^{\text {string }}-T s_{c}-\Delta \varepsilon\right)}{T_{f}^{2} k_{B} \Delta C_{P}\left(T_{f}\right)} \times\left(F_{\text {in }}-\left(T s_{c}^{\text {string }}-T s_{c}-\Delta \varepsilon\right)\right)\right\}
$$

In the limit $T \rightarrow 0$ the distribution of barriers becomes largely independent of temperature with $\Gamma\left(F^{\ddagger}>F_{\text {in }}\right) \sim \exp \left(-a F^{\ddagger}\right)$ and $\alpha \approx\left((z-2) v_{\text {int }}\left(\mathrm{T}_{f}\right)-\Delta \varepsilon\right) /\left(T_{f} k_{B} \Delta C_{P}\left(T_{f}\right)\right)$. For a broad enough distribution of barriers the dielectric absorption spectrum is determined through the simple relation, $\varepsilon^{\prime \prime}(\omega) \sim P\left(F^{\ddagger}=-k_{B} T \ln \omega / \omega_{0}\right) \sim \omega^{a T}$, and becomes flat for low temperatures, resembling the so called constant loss spectrum.

In the inverted non-equilibrium regime, in which an aged system is heated to a temperature above $T_{f}$ (called a rejuvenating glass), the energetic contribution to the driving force is negative $\Delta \varepsilon<0$. In this situation the system appears as if it is further from the dynamical crossover temperature than an equilibrated system at the same temperature and secondary relaxations are relatively suppressed. 
We have shown that by adding fluctuations to the existing structure of random first order transition theory a tail develops on the low free energy side of the activation barrier distribution which shares many of the observed features of the secondary relaxations. The relaxation process responsible for the tail differs from the primary relaxation mechanism in the geometry of the region undergoing cooperative reconfiguration. While primary relaxation takes place through activated events involving compact regions, secondary relaxation is governed by more ramified string-like, or percolation-like clusters of particles. While the existence of secondary relaxation is nearly universally observed in the laboratory, the relevant motions are of shorter length scales than those for primary relaxation, allowing additional material dependent effects and, perhaps, less universal quantitative description than for the main relaxation. The present theory, however, suggests a universal mechanism for secondary relaxation. The theory points out general trends about the way these relaxations vary with temperature and substance which conform to observation.

\section{Acknowledgments}

Support from NSF grant CHE0317017 and NIH grant 5R01GM44557 is gratefully acknowledged. Encouraging discussion on this topic with Vas Lubchenko, Hans Frauenfelder and Jörg Schmalian are gratefully acknowledged

\section{References}

1. Adichtchev S, et al. Fast relaxation processes in glasses as revealed by depolarized light scattering. Journal of Non-Crystalline Solids. 2007; 353:1491-1500.

2. Kudlik A, Benkhof S, Blochowicz T, Tschirwitz C, Rossler E. The dielectric response of simple organic glass formers. J Mol Struct. 1999; 479:201-218.

3. Ngai KL, Capaccioli S. Relation between the activation energy of the Johari-Goldstein $\beta$ relaxation and $T_{g}$ of glass formers. Phys Rev E. 2004; 69:031501.

4. Wang LM, Richert R. Primary and secondary relaxation time dispersions in fragile supercooled liquids. Phys Rev B. 2007; 76:064201.

5. Lunkenheimer P, Schneider U, Brand R, Loidl A. Glassy dynamics. Contemporary Phys. 2000; 41:15-36.

6. Frauenfelder H, et al. A unified model of protein dynamics. Proc Natl Acad Sci U S A. 2009

7. Thayyil MS, Capaccioli S, Prevosto D, Ngai KL. Is the Johari-Goldstein beta-relaxation universal? Philosophical Magazine. 2008; 88:4007-4013.

8. Lubchenko V, Wolynes PG. Theory of structural glasses and supercooled liquids. Ann Rev Phys Chem. 2007; 58:235-266. [PubMed: 17067282]

9. Xia X, Wolynes PG. Fragilities of liquids predicted from the random first order transition theory of glasses. Proc Natl Acad Sci U S A. 2000; 97:2990-2994. [PubMed: 10737779]

10. Stevenson JD, Wolynes PG. Thermodynamic-kinetic correlations in supercooled liquids: A critical survey of experimental data and predictions of the random first-order transition theory of glasses. $\mathrm{J}$ Phys Chem B. 2005; 109:15093-15097. [PubMed: 16852910]

11. Lubchenko V, Wolynes PG. Theory of aging in structural glasses. J Chem Phys. 2004; 121:28522865. [PubMed: 15291595]

12. Xia X, Wolynes PG. Microscopic theory of heterogeneity and nonexponential relaxations in supercooled liquids. Phys Rev Lett. 2001; 86:5526-5529. [PubMed: 11415292]

13. Dzero M, Schmalian J, Wolynes PG. Replica theory for fluctuations of the activation barriers in glassy systems. arXiv:0809.3988v1. 2008

14. Stevenson JD, Schmalian J, Wolynes PG. The shapes of cooperatively rearranging regions in glassforming liquids. Nature Phys. 2006; 2:268-274.

15. Kirkpatrick TR, Thirumalai D, Wolynes PG. Scaling concepts for the dynamics of viscous liquids near an ideal glassy state. Phys Rev A. 1989; 40:1045-1054. [PubMed: 9902230] 
16. Biroli G, Bouchaud JP, Cavagna A, Grigera TS, Verrocchio P. Thermodynamic signature of growing amorphous order in glass-forming liquids. Nature Phys. 2008; 4:771-775.

17. Cammarota C, Cavagna A, Gradenigo G, Grigera TS, Verrocchio P. Surface tension fluctuations and a new spinodal point in glass-forming liquids. arXiv. 2009 0904.1522.

18. Capaccioli S, Ruocco G, Zamponi F. Dynamically correlated regions and configurational entropy in supercooled liquids. J Phys Chem B. 2008; 112:10652-10658. [PubMed: 18671368]

19. Stevenson JD, Walczak AM, Hall RW, Wolynes PG. Constructing explicit magnetic analogies for the dynamics of glass forming liquids. J Chem Phys. 2008; 129:194505. [PubMed: 19026064]

20. Adam G, Gibbs JH. On the temperature dependence of cooperative relaxation properties in glassforming liquids. J Chem Phys. 1965; 43:139-146.

21. Bhattacharyya SM, Bagchi B, Wolynes PG. Facilitation, complexity growth, mode coupling, and activated dynamics in supercooled liquids. Proc Natl Acad Sci U S A. 2008; 105:16077-16082. [PubMed: 18927234]

22. Flory, PJ. Principles of Polymer Chemistry. Cornell University Press Ltd.; 1953.

23. Hagedorn R. Statistical thermodynamics of strong interactions at high-energies. Nuovo Cim Suppl. $1965 ; 3: 147$.

24. Wiedersich J, et al. Fast and slow relaxation processes in glasses. J Phys: Cond Matt. 1999; 11:A147-A156.

25. Döß A, Hinze G, Schiener B, Hemberger J, Böhmer R. Dielectric relaxation in the fragile viscous liquid state of toluene. J Chem Phys. 1997; 107:1740-1743.

26. Plotkin SS, Wolynes PG. Buffed energy landscapes: Another solution to the kinetic paradoxes of protein folding. Proc Natl Acad Sci U S A. 2003; 100:4417-4422. [PubMed: 12677002]

27. Redner, S. A guide to first-passage processes. Cambridge University Press; Cambridge, UK: 2001.

28. Leutheusser E. Dynamical model of the liquid-glass transition. Phys Rev A. 1984; 29:2765-2773.

29. Gotze W, Sjogren L. Relaxation processes in supercooled liquids. Reports On Progress In Phys. 1992; 55:241-376.

30. Singh Y, Stoessel JP, Wolynes PG. Hard-sphere glass and the density-functional theory of aperiodic crystals. Phys Rev Lett. 1985; 54:1059-1062. [PubMed: 10030918]

31. Franz S. Metastable states, relaxation times and free-energy barriers in finite-dimensional glassy systems. Europhys Lett. 2006; 73:492-498.

32. Mezard M, Parisi G. Statistical physics of structural glasses. J Phys: Cond Matt. 2000; 12:66556673.

33. Blochowicz T, Tschirwitz C, Benkhof S, Rossler EA. Susceptibility functions for slow relaxation processes in supercooled liquids and the search for universal relaxation patterns. J Chem Phys. $2003 ; 118: 7544-7555$.

34. Blochowicz T, Gainaru C, Medick P, Tschirwitz C, Rossler EA. The dynamic susceptibility in glass forming molecular liquids: The search for universal relaxation patterns II. J Chem Phys. 2006; 124:134503. [PubMed: 16613457] 


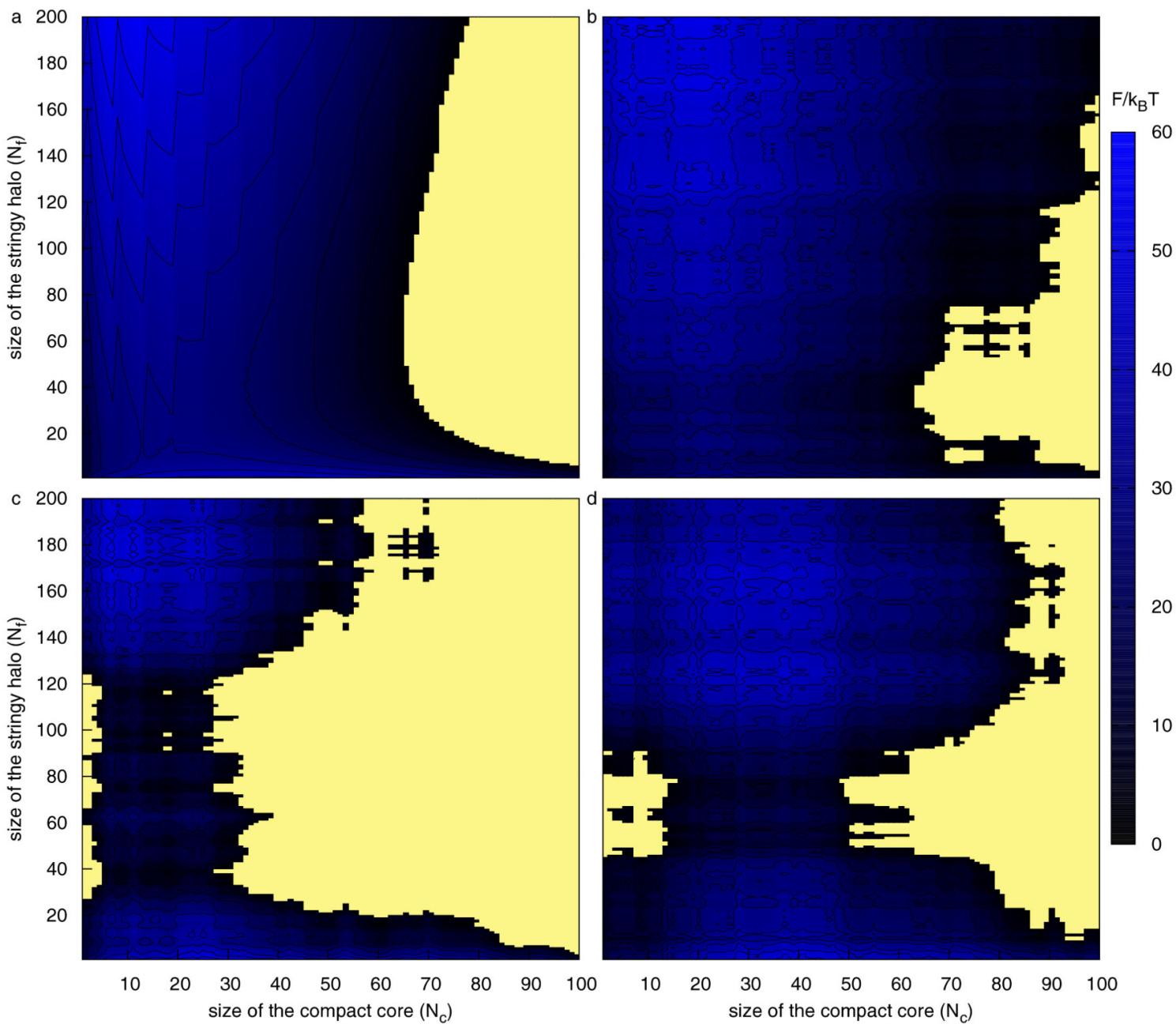

FIG. 1.

Disordered free energy profiles showing the free energy cost of rearranging a region with $N_{c}$ compact particles and $N_{f}$ stringy particles. The transition state separating the unreconfigured state $\left(N_{c}=N_{f}=0\right)$ from the final state where stable reconfigurations are possible (colored yellow) determines the barrier to reconfiguration. The upper left panel shows the fluctuation free profile while the others demonstrate three possible realizations of the fluctuations. In the top right panel situation compact reconfiguration is required to overcome the free energy barrier. For the two other realizations the fluctuations yield stable (yellow) regions along the vertical axis, allowing string-like reconfiguration which account for the secondary relaxation. 


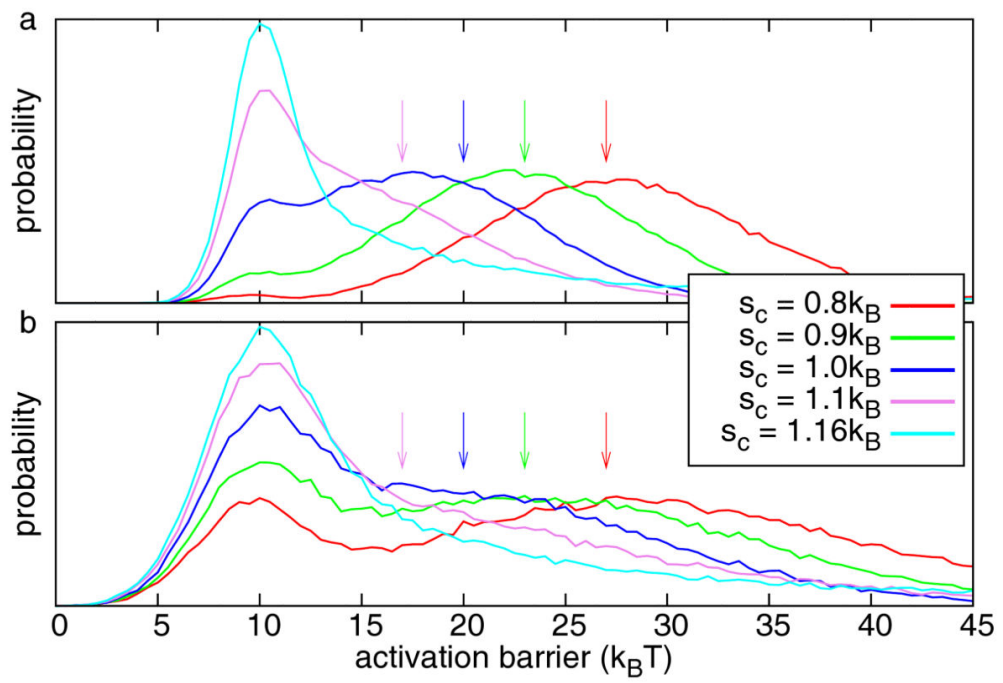

FIG. 2.

Activation barrier distribution for free energy barriers governing relaxation events in supercooled liquids. Different curves represent different temperatures (measured by the configurational entropy $s_{c}$ ), increasing from near the glass transition temperature to just above the dynamical crossover temperature. The arrows indicate the typical relaxation time predicted from the fuzzy sphere model without fluctuations. The top panel corresponds to a rather strong liquid with small fluctuations, $\Delta C_{P} \approx 1 k_{B}$ per bead. The bottom panel corresponds to a fragile liquid with larger fluctuations, $\Delta C_{P}=3 k_{B}$ per bead. In this figure we have used the continuous approximation of $F_{\text {in }}$ as discussed in the text. 

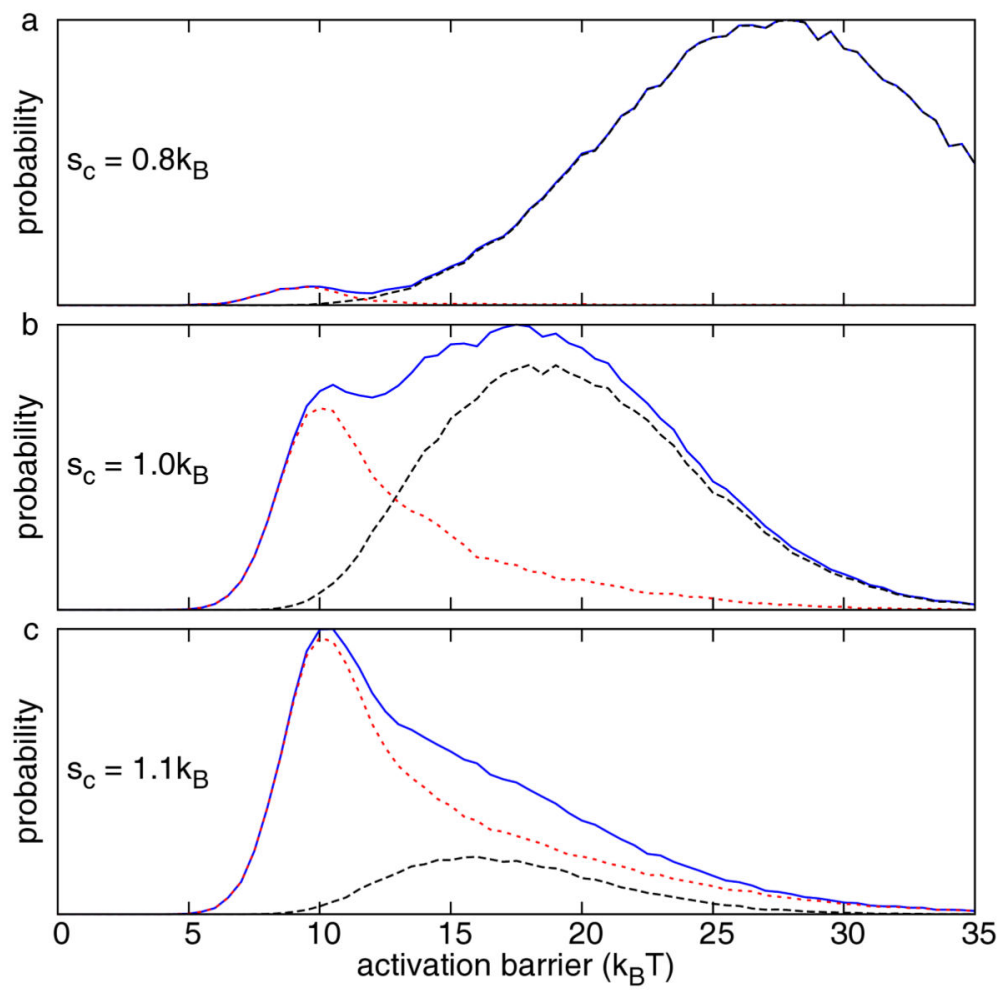

FIG. 3.

Activation barrier distribution for strong liquid with $\Delta C_{P} \approx 1 k_{B}$ per bead, separated into the contribution from secondary relaxations (dotted, red curves), corresponding to string-like reconfiguration as shown in panels $\mathrm{c}$ and $\mathrm{d}$ of figure 1, and primary relaxations (dashed, black curves), corresponding to compact reconfigurations. The full distributions are given for comparison (solid, blue curves). The separation of the curves makes clear that as the dynamical crossover temperature is approached the primary relaxation becomes subordinate to the secondary relaxation. 

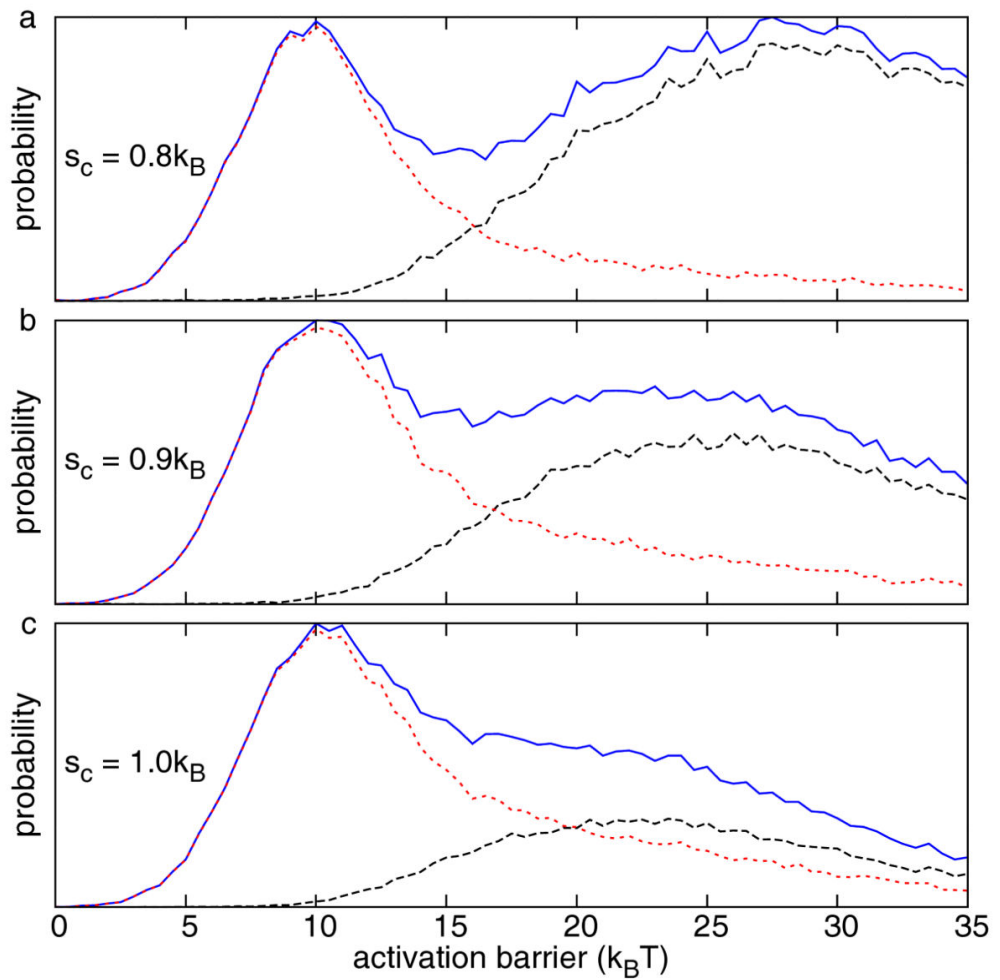

FIG. 4.

Activation barrier distribution for fragile liquids with $\Delta C_{P} \approx 3 k_{B}$ per bead. See figure 3 for details. 


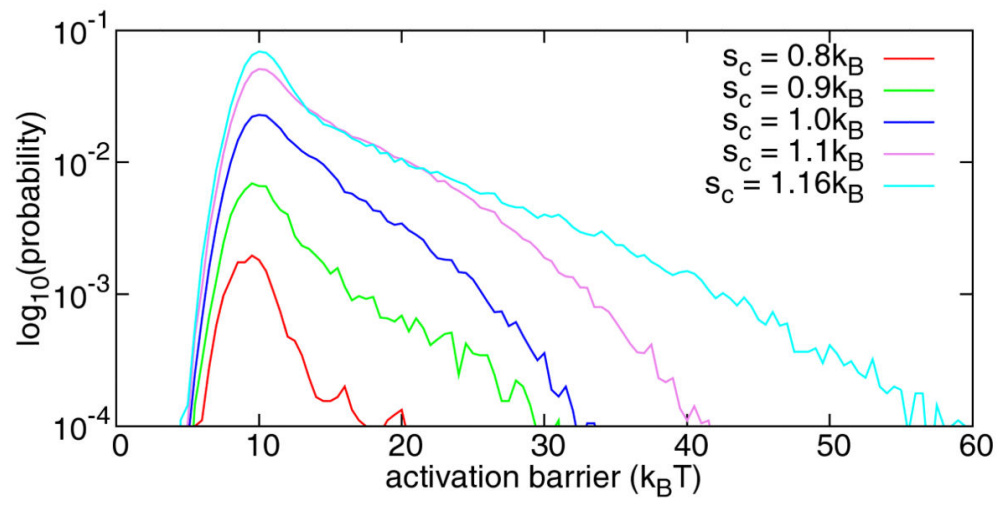

FIG. 5.

Activation barrier distribution for secondary relaxation calculated from the statistical sampling of the fuzzy sphere model with fluctuations. The data correspond to a strong liquid $\left(\Delta C_{P} \approx 1\right)$ and show that at higher temperatures (larger configurational entropies) the distribution decays more slowly. This leads to wider activation energy distributions, matching the expectations of the analytical calculations. 


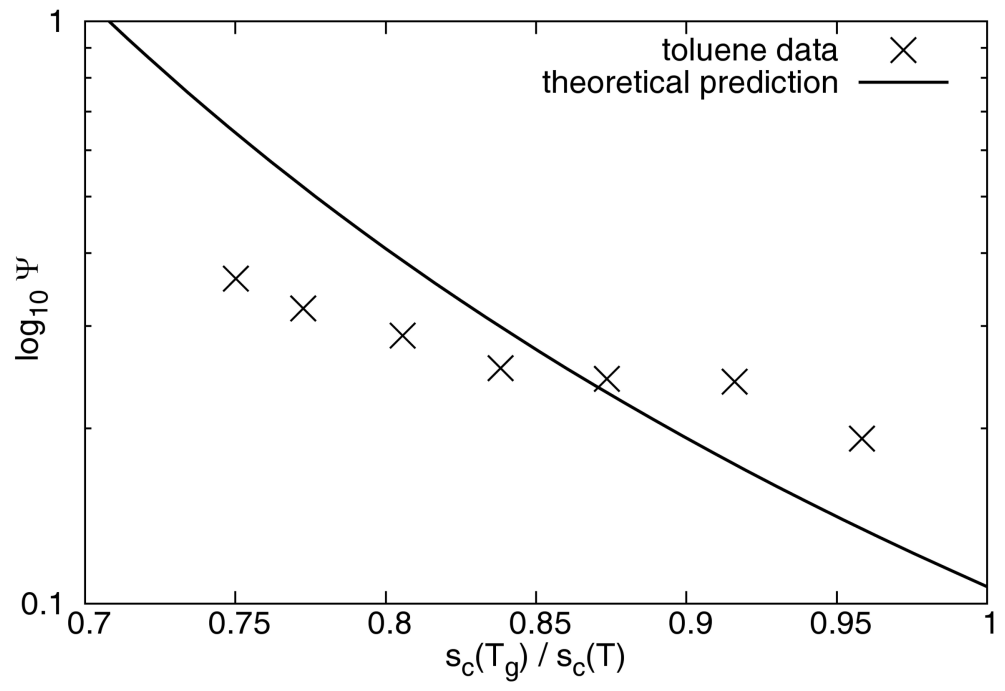

FIG. 6. Relative importance of the secondary process to the total relaxation The analytical theory predicts that secondary relaxations increase in importance with increasing temperature, becoming the dominant process near the dynamical crossover temperature. The experimental data are from reference [24] and plotted as a function of configurational entropy using the relation $s_{c}(T) \approx s_{\infty}\left(1-T_{K} / T\right)$ with $T_{K}$ and $T_{g}$ taken from reference [25]. The magnitude of the driving force fluctuations is estimated from the relation $\delta f \approx T \delta s_{c} \approx T \sqrt{k_{B} \Delta C_{p}}$ with the configurational heat capacity per bead of toluene $\left(\Delta C_{p}=\right.$ $2.9 k_{B}$ ) taken from reference [10]. 